Acta Crystallographica Section D

Biological

Crystallography

ISSN 0907-4449

\title{
High-resolution structure of a retroviral protease folded as a monomer
}

Mason-Pfizer monkey virus (M-PMV), a D-type retrovirus assembling in the cytoplasm, causes simian acquired immunodeficiency syndrome (SAIDS) in rhesus monkeys. Its pepsinlike aspartic protease (retropepsin) is an integral part of the expressed retroviral polyproteins. As in all retroviral life cycles, release and dimerization of the protease (PR) is strictly required for polyprotein processing and virion maturation. Biophysical and NMR studies have indicated that in the absence of substrates or inhibitors M-PMV PR should fold into a stable monomer, but the crystal structure of this protein could not be solved by molecular replacement despite countless attempts. Ultimately, a solution was obtained in $m r$-rosetta using a model constructed by players of the online protein-folding game Foldit. The structure indeed shows a monomeric protein, with the $\mathrm{N}$ - and $\mathrm{C}$-termini completely disordered. On the other hand, the flap loop, which normally gates access to the active site of homodimeric retropepsins, is clearly traceable in the electron density. The flap has an unusual curled shape and a different orientation from both the open and closed states known from dimeric retropepsins. The overall fold of the protein follows the retropepsin canon, but the $\mathrm{C}^{\alpha}$ deviations are large and the active-site 'DTG' loop (here NTG) deviates up to $2.7 \AA$ from the standard conformation. This structure of a monomeric retropepsin determined at high resolution $(1.6 \AA)$ provides important extra information for the design of dimerization inhibitors that might be developed as drugs for the treatment of retroviral infections, including AIDS.

\section{Introduction}

Mason-Pfizer monkey virus (M-PMV), or simian retrovirus type 3 (SRV-3), is a D-type retrovirus (assembling in the cytoplasm of the infected cell) that causes simian acquired immunodeficiency syndrome (SAIDS) in Asian monkeys of the genus Macaca. Its protease (PR), which is necessary for the processing of, but is also an integral part of, the expressed retroviral fusion polyproteins, is autocatalytically excised as a $17 \mathrm{kDa}$ form that undergoes further C-terminal processing to a $13 \mathrm{kDa}$ (13PR) form. Protease activation and Gag processing must be highly regulated in M-PMV, since the PR remains inactive as part of the Gag-Pro and Gag-Pro-Pol polyproteins until a late stage of virus release from the cell. The C-terminal part, which contains a glycine-rich region called the G-patch (Bauerová-Zábranská et al., 2005; Svec et al., 2004), is not
Received 16 August 2011 Accepted 3 September 2011

PDB Reference: monomeric M-PMV retroviral protease, 3sqf. 
necessary for PR activity but is indispensable for the activity of reverse transcriptase (RT) and for virus infectivity, and most probably functions as the $\mathrm{N}$-terminus of RT after the proteolytic cleavage of the Gag-Pro-Pol polyprotein (Krizova et al., unpublished results). In vitro, C-terminal autoprocessing of $13 \mathrm{PR}$ proceeds even further, yielding a $12 \mathrm{kDa}$ (12PR) form of reduced activity (Zábranský et al., 1998). M-PMV (and also HIV) PR is activated under reducing conditions in a process that is likely to involve Cys residues in the retroviral Gag polyprotein. In its active form, retroviral PR is a pepsin-like homodimeric enzyme (retropepsin) with an active site composed of two DTG loops, each contributing one aspartate to a water-molecule-bound nucleophilic element (Wlodawer et al., 1989). The integrity of a retropepsin homodimer is maintained by a $\beta$-sheet interface woven from alternating $\mathrm{N}$-termini and C-termini of the subunits, with additional contacts contributed by two flexible flap loops and the catalytic triads themselves.

Since the elucidation of its structure, HIV-1 PR has become the most studied target for rational drug design; indeed, there are now ten PR inhibitors that are used in the clinical treatment of AIDS, which act as substrate analogues blocking the active site of the enzyme. However, the emergence of drugresistant mutants calls for alternative strategies; the disruption of PR dimerization would be an attractive possibility (Koh et $a l ., 2007)$ as it would not interfere with the functioning of host aspartic proteases, which are single-chain proteins. However, this potential drug-design approach has so far been unsuccessful. Therefore, systems such as M-PMV PR, in which the regulation of PR activity is important for virus replication and has been better studied, might benefit efforts aimed at inhibiting HIV-1 PR dimerization and the development of a new generation of drugs for the treatment of AIDS. Indeed, biophysical experiments have indicated that $\mathrm{M}$ PMV 13PR should form a monomer-dominated equilibrium (shifted towards the dimer in the presence of substrate/inhibitor), in agreement with the NMR structure of the 12PR variant (Veverka et al., 2003).

In the present study, we used a $13 \mathrm{PR}$ protein (Trp1-Ala114) with C7A/C106A/ D26N mutations. The Cys $\rightarrow$ Ala substitutions remove the possibility of uncontrolled S-S aggregation and mimic the Cys-activated $\mathrm{PR}$ in vivo. The $\mathrm{D} 26 \mathrm{~N}$ substitution changes the PR active site DTG triplet to prevent autodigestion. The protein could be crystallized in several crystal forms. Some of the crystals were obtained in the presence of an inhibitor added as a dimerization 'bait' with the intention of making the crystal structure amenable to molecular-replacement (MR) methods. The best crystals (monoclinic $P 2_{1}$ ), used in this study, with an estimated two protein molecules in the asymmetric unit, were grown in the presence of a 1.2-fold molar excess of a peptidomimetic inhibitor. However, the crystal structure resisted all MR attempts, which utilized all available programs and existing crystallographic models of retropepsins (full dimers and individual subunits). The NMR model of monomeric 12PR could also not be used to solve the crystal structure. The mr-rosetta algorithm, which has an outstanding record of success with difficult structures, also failed to produce a solution using the existing models (DiMaio et al., 2011). This daunting protein-folding problem was therefore presented as a challenge to Foldit (Cooper et al., 2010) players, who generated over one million models starting from the NMR coordinates. One of these solutions, when submitted to MR calculations in $m r$-rosetta (DiMaio et al., 2011), did produce a plausible crystal structure (Khatib et al., 2011) that could be easily refined to an $R$ factor of 0.169 with excellent geometry. The details of the success of the FolditRosetta approach using a computer-game-derived model have been described elsewhere (Khatib et al., 2011).

\section{Materials and methods}

\subsection{Cloning, expression and purification of $\mathrm{C} 7 \mathrm{~A} / \mathrm{C} 106 \mathrm{~A} /$ D26N 13PR}

The mutations were introduced into the previously described plasmid pBPS13ATG using the QuikChange SiteDirected Mutagenesis Kit (Stratagene; Zábranská et al., 2007) and verified by DNA sequencing. The expression of M-PMV PR was carried out in Escherichia coli BL21 (DE3) cells under previously described conditions (Zábranská et al., 2007). The protease, which was expressed in inclusion bodies, was
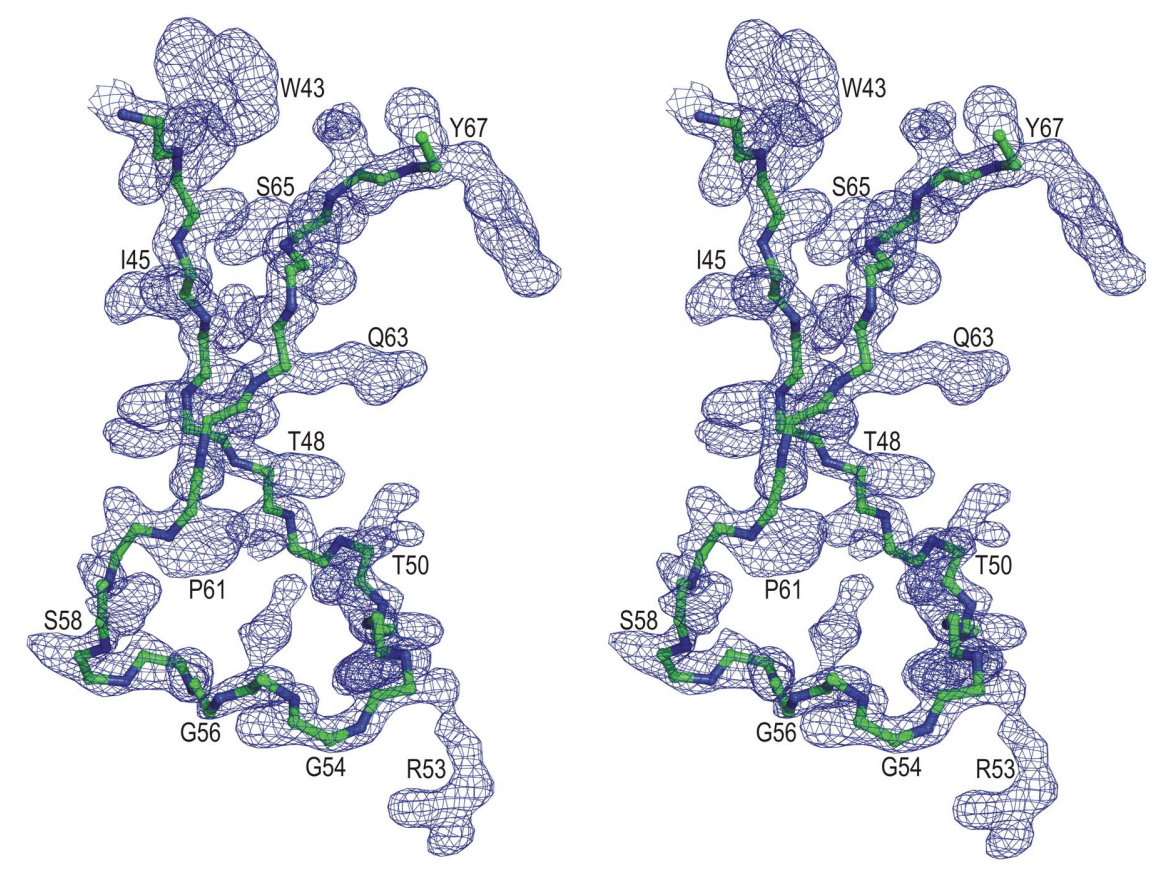

Figure 1

Stereoview of the main-chain trace of the flap loop plus flanking residues (Trp43-Tyr67). This trace of the flap of molecule $A$ is shown in $2 F_{\mathrm{o}}-F_{\mathrm{c}}$ electron density contoured at $1.0 \sigma$. Sidechain atoms have been omitted for clarity. 
Table 1

Data-collection and structure-refinement statistics.

Values in parentheses are for the highest resolution shell.

\begin{tabular}{|c|c|}
\hline \multicolumn{2}{|l|}{ Data collection } \\
\hline Crystal dimensions (mm) & $0.30 \times 0.15 \times 0.15$ \\
\hline Space group & $P 2_{1}$ \\
\hline Unit-cell parameters $\left(\AA{ }^{\circ},{ }^{\circ}\right)$ & $\begin{array}{c}a=26.76, b=86.62 \\
\quad c=39.31, \beta=104.6\end{array}$ \\
\hline Solvent content (\%) & 28.1 \\
\hline Temperature (K) & 100 \\
\hline $\mathrm{X}$-ray source & EMBL/DESY X13 \\
\hline Wavelength $(\AA ̊)$ & 0.8086 \\
\hline Oscillation angle $\left({ }^{\circ}\right)$ & 0.5 \\
\hline No. of frames & 456 \\
\hline Resolution $(\AA)$ & $43.3-1.63(1.73-1.63)$ \\
\hline Mosaicity $\left({ }^{\circ}\right)$ & 0.28 \\
\hline$R_{\text {int }}^{\dagger}$ & $0.068(0.752)$ \\
\hline$R_{\text {meas }} \ddagger$ & $0.076(0.860)$ \\
\hline$\langle I / \sigma(I)\rangle$ & $14.9(1.9)$ \\
\hline \multicolumn{2}{|l|}{ Reflections } \\
\hline Measured & 99683 \\
\hline Unique & 21369 \\
\hline Completeness (\%) & $99.0(96.3)$ \\
\hline Multiplicity & $4.7(4.2)$ \\
\hline Wilson $B$ factor $\left(\AA^{2}\right)$ & 26.0 \\
\hline \multicolumn{2}{|l|}{ Refinement } \\
\hline Resolution (£) & $28.6-1.63$ \\
\hline \multicolumn{2}{|l|}{ No. of reflections } \\
\hline Work set & 20295 \\
\hline Test set & 1070 \\
\hline$R / R_{\text {free }} \S$ & $0.1694 / 0.2124$ \\
\hline Protein molecules in asymmetric unit & 2 \\
\hline \multicolumn{2}{|l|}{ No. of atoms } \\
\hline Protein & 1527 \\
\hline Water & 154 \\
\hline \multicolumn{2}{|l|}{$\langle B$ factor $\rangle\left(\AA^{2}\right)$} \\
\hline Protein & 28.4 \\
\hline Water & 34.6 \\
\hline \multicolumn{2}{|l|}{ R.m.s. deviations from ideal } \\
\hline Bond lengths $(\AA)$ & 0.018 \\
\hline Bond angles $\left({ }^{\circ}\right)$ & 1.77 \\
\hline \multicolumn{2}{|l|}{ Ramachandran statistics (\%) } \\
\hline Favoured & 96.8 \\
\hline Allowed & 3.2 \\
\hline Outliers & 0.0 \\
\hline PDB code & $3 \mathrm{sqf}$ \\
\hline
\end{tabular}

$\dagger R_{\text {int }}=\sum_{h k l} \sum_{i}\left|I_{i}(h k l)-\langle I(h k l)\rangle\right| / \sum_{h k l} \sum_{i} I_{i}(h k l)$, where $I_{i}(h k l)$ is the $i$ th measurement of the intensity of reflection $h k l$ and $\langle I(h k l)\rangle$ is the mean intensity of reflection $h k l . \quad$ 末 $R_{\text {meas }}=\sum_{h k l}[N /(N-1)]^{1 / 2} \sum_{i}\left|I_{i}(h k l)-\langle I(h k l)\rangle\right| / \sum_{h k \mid} \sum_{i} I_{i}(h k l)$, where $I_{i}(h k l)$ is the $i$ th measurement of the intensity of reflection $h k l,\langle I(h k l)\rangle$ is the mean intensity of reflection $h k l$ and $N$ is the number of observations of intensity $I(h k l)$ (multiplicity). $§ R=\sum_{h k l}|| F_{\text {obs }}|-| F_{\text {calc }}|| / \sum_{h k l}\left|F_{\text {obs }}\right|$, where $F_{\text {obs }}$ and $F_{\text {calc }}$ are the observed and calculated structure factors, respectively. $R_{\text {free }}$ was calculated analogously for a randomly selected $5 \%$ of the reflections.

renatured by solubilization in $8 M$ urea and stepwise dialysis against $50 \mathrm{~m} M$ Tris- $\mathrm{HCl} \mathrm{pH} 7.0,1 \mathrm{~m} M$ EDTA, $0.05 \%$ $\beta$-mercaptoethanol (buffer $A$ ) and was purified by ionexchange chromatography (batch method) on QAE-Sephadex A-25 equilibrated with buffer $A$.

\subsection{Crystallization}

Prior to crystallization experiments, the protein was incubated overnight with a 1.2-fold molar excess (relative to dimeric enzyme) of a peptidomimetic inhibitor with the sequence Pro-Tyr-Val-Pst-Ala-Met-Thr, where Pst is $(3 S, 4 S)$ - 4-amino-3-hydroxy-5-phenylpentanoic acid, and a $K_{\mathrm{i}}$ of $5.3 \mathrm{n} M$ for wt 13PR protein. Crystallization screens were set up manually using Crystal Screen and Crystal Screen 2 (Hampton Research; Jancarik \& Kim, 1991) and the hangingdrop vapour-diffusion technique at $292 \mathrm{~K}$ by mixing $1 \mu \mathrm{l}$ protein solution $\left(8.5 \mathrm{mg} \mathrm{ml}^{-1}\right.$ in $10 \mathrm{mM}$ Tris $\mathrm{pH} \mathrm{8.5)}$ and $1 \mu \mathrm{l}$ reservoir solution. Crystals grew to dimensions of $0.3 \times 0.15 \times$ $0.15 \mathrm{~mm}$ within two weeks over a reservoir solution consisting of $0.1 M$ imidazole $\mathrm{pH} 6.5$ and $1 M$ sodium acetate. For cryoprotection, the crystal was transferred to a solution consisting of the crystallization mother liquor supplemented with $15 \%(v / v)$ glycerol.

\subsection{Data collection and processing}

X-ray diffraction data were collected at $100 \mathrm{~K}$ on a MAR CCD $165 \mathrm{~mm}$ detector system using synchrotron radiation on EMBL/DESY (Hamburg) beamline X13. Integration, scaling and merging of the intensity data was carried out in the $X D S$ package (Kabsch, 2010). The unit-cell parameters and Bravais lattice were determined using the COLSPOT and $I D X R E F$ subroutines in $X D S$. The intensities were reduced to structure-factor amplitudes by the method of French \& Wilson (1978) and then converted to MTZ format using the F2MTZ and $C A D$ routines of $C C P 4$ (Winn et al., 2011). Space group, unit-cell and data-collection parameters are summarized in Table 1.

\subsection{Structure solution and refinement}

A model generated by Foldit (Cooper et al., 2010) players (Khatib et al., 2011) from the NMR coordinates 1nso (Veverka et al., 2003) successfully solved the structure in mr-rosetta (DiMaio et al., 2011). An initial atomic model of the structure was autobuilt and refined in PHENIX (Adams et al., 2010). Manual rebuilding of the model and divining of water molecules was performed in Coot (Emsley \& Cowtan, 2004). Maximum-likelihood structure refinement was carried out in PHENIX (Adams et al., 2010) using all intensity data, with the exception of 1070 reflections (5\%) flagged for cross-validation purposes. No $\sigma$ cutoff was applied. Successive rounds of manual rebuilding and refinement of the initial model resulted in $R$ and $R_{\text {free }}$ values of 0.2715 and 0.2786 , respectively. The next ten cycles of simulated-annealing refinement in phenix.refine lowered the $R$ factor to 0.2300 . Implementation of TLS parameters, selected according to the TLSMD server (Painter $\&$ Merritt, 2005), and addition of $\mathrm{H}$ atoms at riding positions as a fixed contribution to $F_{\mathrm{c}}$ lowered the $R$ factor below 0.2 . Optimization of X-ray/stereochemistry weighting in PHENIX, refinement of the occupancies of some water molecules and several rounds of manual modelling resulted in final $R$ and $R_{\text {free }}$ values of 0.1694 and 0.2124 , respectively. The final model consisted of residues 9-103 of chain $A$, residues 9-102 of chain $B$ and 154 water molecules. The refinement statistics are given in Table 1. Structural illustrations were prepared with $P y M O L$ (DeLano, 2002). 
Table 2

R.m.s.d. values ( $\AA$ ) for core $\mathrm{C}^{\alpha}$ superpositions of molecule $A$ of M-PMV PR on molecule $B$ and on protomers of aspartic retroviral proteases, N- and Cterminal domains of porcine pepsin and the retropepsin-like putative protease domain of the eukaryotic protein Ddi1 (PDB codes are given in parentheses).

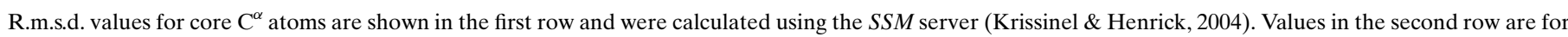

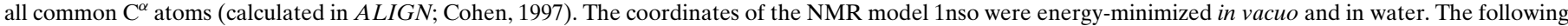

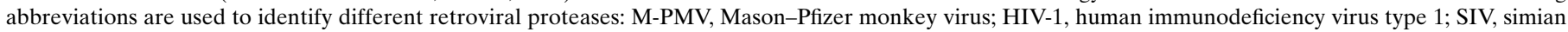

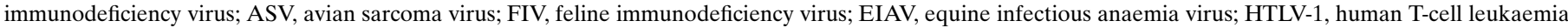
virus type 1; XMRV, xenotropic murine leukaemia virus-related virus.

\begin{tabular}{|c|c|c|c|c|c|c|c|c|c|c|c|c|c|c|}
\hline \multicolumn{4}{|c|}{ M-PMV } & \multirow[b]{3}{*}{$\begin{array}{l}\text { HIV-1 } \\
(3 \mathrm{hvp}) \dagger\end{array}$} & \multirow[b]{3}{*}{$\begin{array}{l}\text { HIV-1 } \\
(4 h v p) \neq\end{array}$} & \multirow[b]{3}{*}{$\begin{array}{l}\text { SIV } \\
(1 y \text { th }) \neq\end{array}$} & \multirow[b]{3}{*}{$\begin{array}{l}\text { ASV } \\
(2 \mathrm{rsp}) \dagger \S\end{array}$} & \multirow[b]{3}{*}{$\begin{array}{l}\text { FIV } \\
(4 \text { fiv }) \ddagger\end{array}$} & \multirow[b]{3}{*}{$\begin{array}{l}\text { EIAV } \\
(2 \mathrm{fmb}) \ddagger\end{array}$} & \multirow[b]{3}{*}{$\begin{array}{l}\text { HTLV-1 } \\
\text { (3liy) } \ddagger\end{array}$} & \multirow[b]{3}{*}{$\begin{array}{l}\text { XMRV } \\
(3 n r 6) \dagger\end{array}$} & \multirow[b]{3}{*}{$\begin{array}{l}\text { Ddi1 } \\
(2 \mathrm{i} 1 \mathrm{a})\end{array}$} & \multirow{2}{*}{\multicolumn{2}{|c|}{ Pepsin (4pep) }} \\
\hline & & NMR ( & so) & & & & & & & & & & & \\
\hline$B$ & Foldit & Vacuo & Water & & & & & & & & & & $\mathrm{N}$ & $\mathrm{C}$ \\
\hline 0.18 & 2.08 & 3.04 & 2.57 & 2.14 & 2.17 & 2.09 & 1.54 & 1.94 & 1.65 & 2.05 & 1.95 & 2.23 & 4.17 & 3.13 \\
\hline 0.18 & 2.87 & 5.51 & 4.33 & 8.92 & 9.06 & 7.73 & 7.95 & 4.28 & 8.23 & 3.50 & 10.49 & 9.77 & 4.44 & 5.93 \\
\hline
\end{tabular}

$\dagger$ Apo form. $\ddagger$ Inhibitor complex. $\S$ Flap loops missing.

\section{Results and discussion}

\subsection{Overall characteristics of the crystal structure}

Despite its use during crystallization, the inhibitor is not present in the crystal structure and the protein exists in a monomeric fold. There are two independent 13PR molecules ( $A$ and $B)$ in the asymmetric unit. They are virtually identical $\left(\mathrm{C}^{\alpha}\right.$ r.m.s.d. of $0.18 \AA$ ) and have the general chain topology known from the structures of dimeric retropepsins (Miller et al., 1989; Wlodawer et al., 1989). The polypeptide chains have excellent electron density for all structural elements, except for the $\mathrm{N}$-terminus (residues 1-8) and C-terminus (104-114). The residues forming the flap loops show increased mobility (especially at the tips; Gln57Ser58), which is visible as higher $B$ factors, but there is no ambiguity about the tracing of these loops (Fig. 1) and their identical conformation in both molecules.

\subsection{Conformation of the M-PMV PR monomer}

The secondary structure assigned using DSSP (Kabsch \& Sander, 1983) illustrates that the pseudo-twofold symmetry noted earlier in the protomers of retroviral proteases (Miller et al., 1989) is preserved quite well in M-PMV PR. Notably, there is a helical

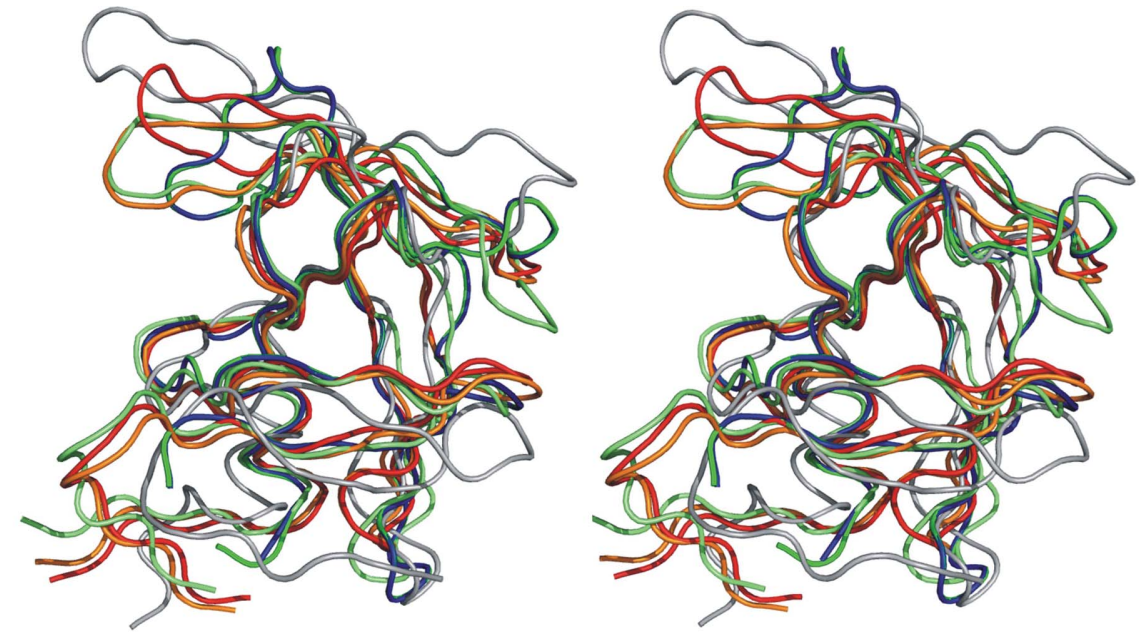

(a)

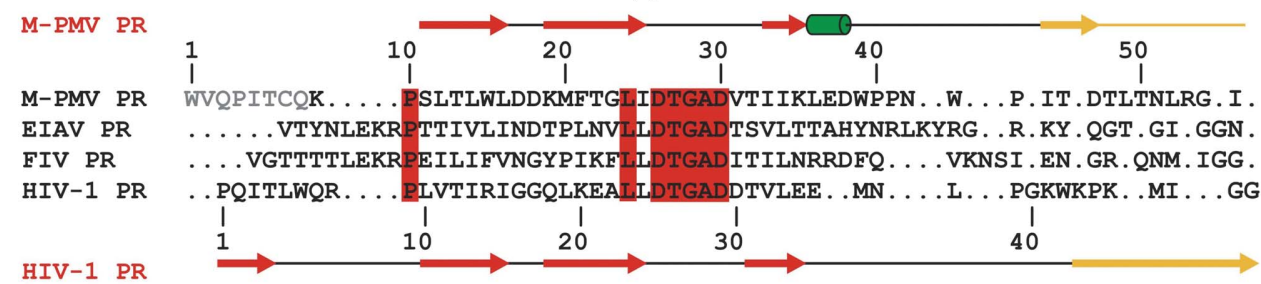

M-PMV PR

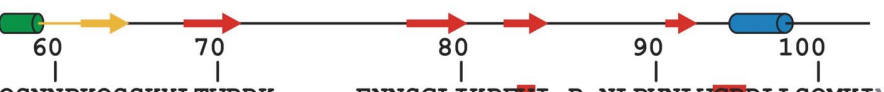

110

M-PMV PR ...GQSNNPKQSSKYLTWRDK. . . . ENNSGLIKPFVI . P. NLPVNLWGRDLLSQMKIMMCSPNDIVTA

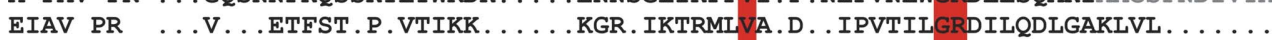
FIV PR ...K...RGTNY. INVHLEIRDENYKT.QCIFGNVCVLEDNSLIQPLLGRDNMIKFNIRLVM . . . . . HIV-1 PR IGGF. . IKVRQY.DQIPVEIC . . . . G. HKAIGTVLVG. P. . TPVNIIGRNLLTQIGCTLNF . . . . . . HIV-1 PR

(b)

Figure 2

Alignment of retroviral proteases. (a) Stereoview of the superposition of the $\mathrm{C}^{\alpha}$ traces of protomers of retroviral proteases: green and blue, M-PMV ( $A$ and $B$ ); red, HIV-1, apo form (PDB entry 3hvp); orange, HIV-1, inhibitor complex (PDB entry 4hvp); lime, EIAV (PDB entry 2fmb); grey, M-PMV, NMR model (PDB entry 1nso), energy-minimized (in water). (b) Structure-based sequence alignment of the M-PMV, EIAV (PDB entry $2 \mathrm{fmb}$; lowest core $\mathrm{C}^{\alpha}$ r.m.s.d.; Table 2), FIV (PDB entry 4 fiv; highest level of sequence identity $-26.6 \%$ ) and HIV-1 (PDB entry 3hvp) proteases. Residue numbers and secondary-structure elements (arrows, $\beta$-strands; blue, $\alpha$-helices; green, $3_{10}$-helices; yellow, flap loops) are marked for the M-PMV and HIV-1 proteases. Residues that are identical in all four sequences are shown on a red background. Disordered residues missing from the M-PMV PR structure are shown in grey. 

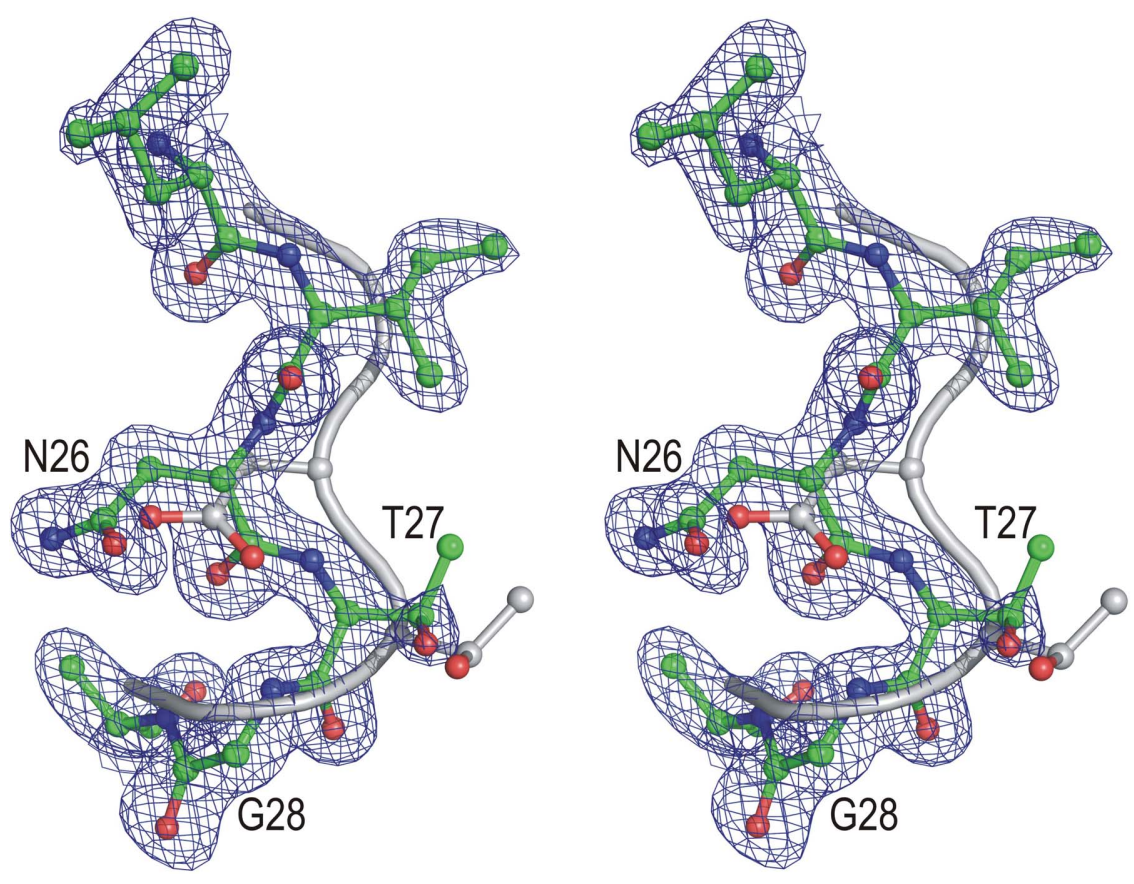

Figure 3

Stereoview of overlay of the active-site (D/N)TG loops of HIV-1 PR (PDB entry 3hvp, grey) and M-PMV PR (green) based on $\mathrm{C}^{\alpha}$ superposition of the entire molecules. The M-PMV PR structure is shown as $2 F_{\mathrm{o}}-F_{\mathrm{c}}$ electron density contoured at $1.3 \sigma$.

segment present in the N-terminal half of the protein (Leu36Asp38), a feature that replicates the canonical C-terminal helix (Arg95-Leu98) but which so far has only been found in EIAV (equine infectious anaemia virus) PR (Kervinen et al., 1998). The C-terminal $\alpha$-helix, however, is shorter than in most retropepsins.

\subsection{The flap loop}

The flap of M-PMV PR (residues Ile45-Ser64; Fig. 2b) has a peculiar shape. It is not a smooth hairpin with $\beta$-type interactions as in other retropepsins, but has a wide conformation with a 310 -helical segment (Gln57-Asn59) present in its C-terminal part. The flap folds upon the body of the protein but in a way that is different from the 'lowered' flap position over the active site of retropepsin dimers in complex with inhibitors (Fig. 2a). The flap arm appears to be much shorter because of the helical insertion and its blunt end. The leading/ trailing strands follow the 'lowered'/'open' flap traces of HIV$1 \mathrm{PR}$. The $3_{10}$-helix in the trailing strand resembles a helical insertion in the flap of HTLV-1 (human T-cell leukaemia virus type-1) PR (Li et al., 2005).

\subsection{The active-site loop}

The active-site loop with the DTG (here NTG) triad has the general conformation as in other pepsins. However, in the absence of its replica, the key interactions $\left(\mathrm{O}^{\delta 1} \cdots\right.$ Wat $\cdots \mathrm{O}^{\delta 1}$, 'fireman's grip') are missing and the side chains of Asn26 and Thr27 form only weak ( $3 \AA$ ) contacts with water molecules. On close comparison, the loop deviates significantly from the trace in HIV-1 PR (Fig. 3); the $\mathrm{C}^{\alpha}$ deviations culminate (2.1 ) at Asn26, with the departure of the $\mathrm{O}^{\delta 1}$ atom being even larger $(2.7 \AA)$. This indicates that fine-tuning of the active-site geometry of retropepsins is only possible upon dimerization.

\subsection{Comparison with other models of retropepsins}

In $\mathrm{C}^{\alpha}$ superpositions, the monomer of $\mathrm{M}$ PMV PR shows marked departures from the subunit folds of dimeric retropepsin structures in the PDB (Table 2), with the most pronounced differences seen in the flap region. The core $\mathrm{C}^{\alpha}$ atoms have r.m.s. deviations of $\sim 2 \AA$, but when all $\mathrm{C}^{\alpha}$ atoms are included the deviations are much larger ( $\geq 3.5 \AA$ ), explaining the failure of the MR calculations. The ASV (avian sarcoma virus) PR model 2rsp (Jaskólski et al., 1990) has an artificially low r.m.s.d. value (1.54 ̊) because of its missing flaps. Of all the retropepsin protomers (as well as homologous proteins and domains; Table 2), the closest structural homologue is the protein from HTLV-1, but the best agreement in the core region is with EIAV PR. The Foldit model used to solve the structure by MR (Khatib et al., 2011) has a similar core r.m.s.d. as the crystallographic models of retropepsins but the value calculated for all $\mathrm{C}^{\alpha}$ atoms is significantly improved, reflecting inter alia that the flap has a generally correct conformation.

On the background of the numerous superpositions with crystallographic models of retropepsins (Fig. $2 a$ ), the similarity to the NMR model of M-PMV 12PR in the core region is the lowest. Here again the flap shows a widely different conformation, but even with its exclusion the match of the protein core is inferior. The alignments reported in Table 2 were calculated for two energy-minimized models of the NMR coordinates 1nso kindly provided by Dr Richard Hrabal. These results explain why the NMR structure 1nso failed to solve the crystal structure directly as an MR model. Incidentally, a similar r.m.s.d. value is obtained for the only other NMR structure of a retroviral protease (from simian foamy virus) monomer in the PDB (PDB entry 2jys; Hartl et al., 2008).

\subsection{Structural consequences of the monomeric fold}

There is no question about the absence of proper biologically competent dimers of the protease in this crystal structure because the $\mathrm{N}$ - and $\mathrm{C}$-terminal peptides, which are absolutely required for and highly ordered upon dimerization, are totally disordered. The disordered fragments include the cysteine residues Cys7 and Cys106 (here mutated to Ala) which are known to connect the termini under nonreducing conditions. The existence of the Cys7-Cys106 bond has been demon- 


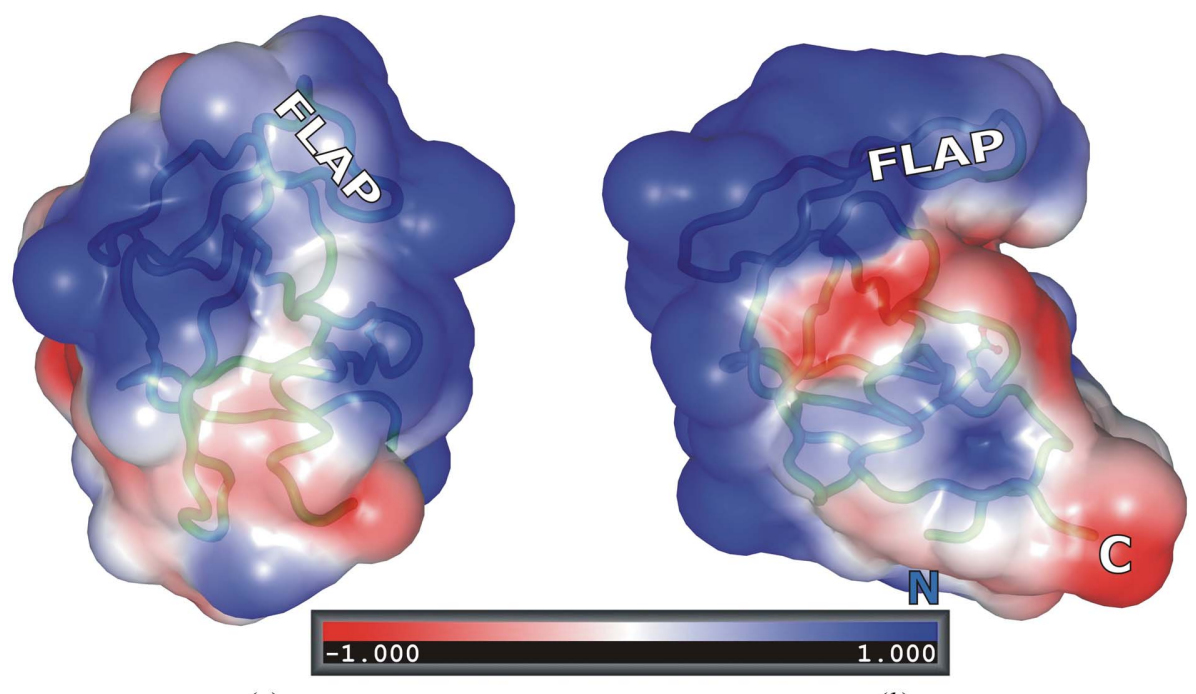

(a)

(b)

Figure 4

Electrostatic potential surface of retroviral protease protomers. The M-PMV PR monomer $(a)$ is shown in the same orientation and on the same scale as the HIV-1 PR protomer (b) extracted from the dimeric molecule (PDB entry $3 \mathrm{hvp}$ ). The complete HIV-1 PR dimer is generated by the action of a vertical dyad, which creates a second copy facing the first molecule on the right. In this view, the $\mathrm{N}$ - and C-termini (missing in M-PMV PR) are at the bottom and the flap loops are at the top. The active-site cavity is marked by the Asn26/Asp25 residue (ball-and-stick representation). In M-PMV PR the cavity is completely covered by the curled flap. The area of positive potential on this M-PMV PR surface is influenced by the D26N substitution, but it is of note that this mutation does not influence the tendency of the protein to fold as a monomer. The electrostatic potential (negative, red; positive, blue) was calculated in APBS (Baker et al., 2001).
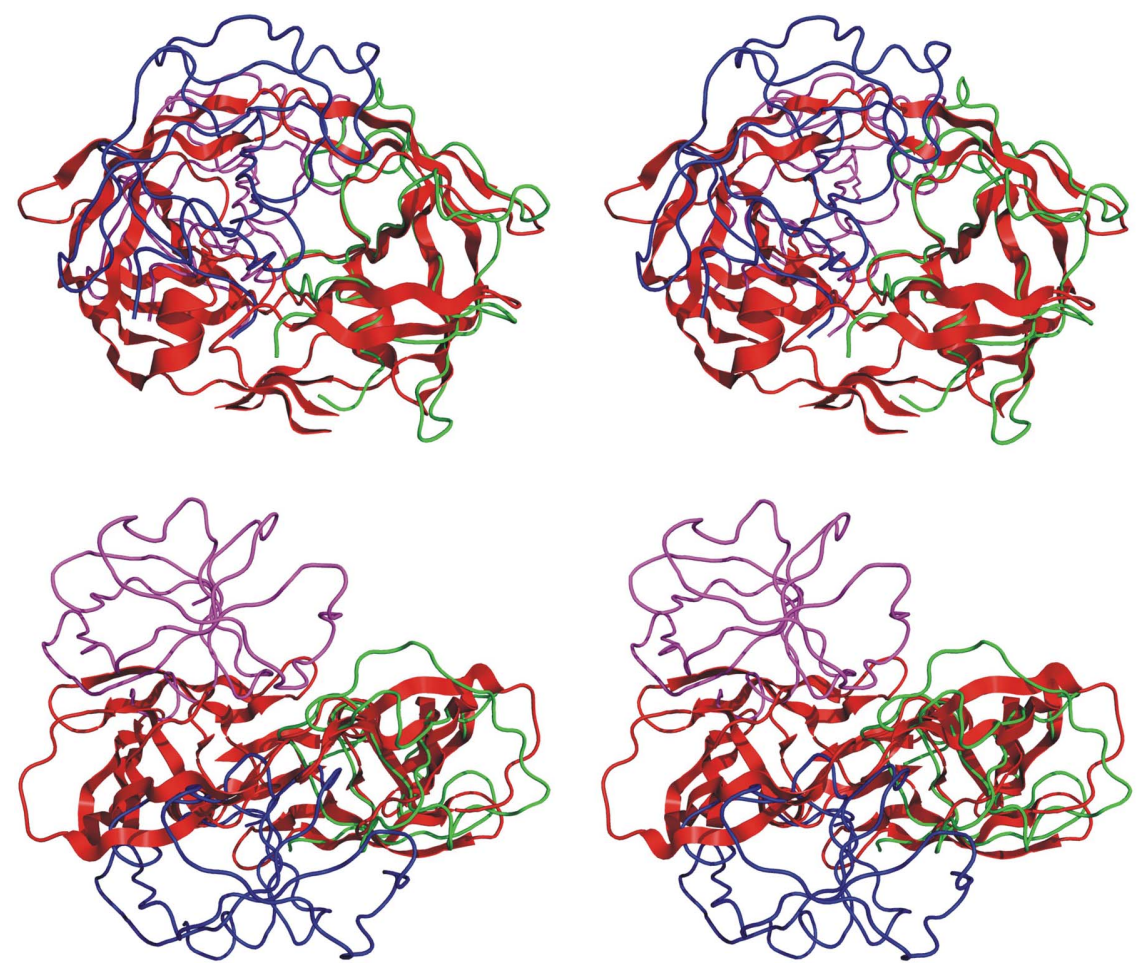

Figure 5

Stereoview of superposition of the $\mathrm{C}^{\alpha}$ atoms of the HIV-1 PR protomer (PDB entry 3hvp, red) on monomer $A$ of M-PMV PR in the crystal structure (green). This superposition illustrates the relation of the HIV-1 PR dimer (cartoon) to the neighbouring copies of M-PMV PR monomer $B$ in the crystal (blue and magenta). Bottom panel, view down the twofold axis of the HIV-1 PR dimer; top panel, a perpendicular view with the twofold axis vertical. strated in monomeric M-PMV PR, but it can be envisaged that it could be reconfigured into an intermolecular context upon dimerization, as the canonical topology of the dimeric interface is $\mathrm{N}(A)-\mathrm{C}(B)-\mathrm{C}(A)-\mathrm{N}(B)$. The novel type of interface reported recently for XMRV (xenotropic murine leukaemia virus-related virus) PR ( $\mathrm{Li}$ et al., 2011) is not applicable in this case as it does not include the N-terminal peptide at all. In the intramolecular context, the Cys7-Cys106 disulfide stabilizes the monomeric fold, while in the intermolecular context it would be expected to reinforce the dimer. Indeed, it has been shown that in the C7A/ C106A mutant the enzymatic activity is reduced in vitro by $60 \%$ (Zábranská et al., 2007). However, in vivo these mutations do not influence Gag processing and virus infectivity. Since the reversible oxidation of immature M-PMV particles has been shown to regulate $\mathrm{PR}$ activation in vitro (Parker \& Hunter, 2001), one can speculate that other cysteines in the Gag polyproteins also participate in PR activation by modulating the conformation and accessibility of the PR cleavage sites or by regulating the binding of cellular proteins that could protect the polyproteins from premature processing.

When the present M-PMV PR molecule is viewed from the direction of its absent dimerization partner, one sees a uniformly positively charged surface (Fig. 4). This is different from a similar view of the HIV-1 PR protomer, in which both charges and hydrophobic patches are seen, and may partly explain why in the absence of substrate/ inhibitor the M-PMV protein can stably exist as a monomer, at least with the D26N mutation. Fig. 4 also illustrates that the curled flap closely covers the active-site cavity, while in the HIV-1 PR protomer extracted from the dimeric enzyme the cavity would be freely accessible.

\subsection{Crystal packing and molecular interactions}

The crystal packing is very dense, with only $28.1 \%$ of the unit-cell volume occupied by solvent (Table 1). Despite 
this, the two protein molecules in the asymmetric unit do not form a tight intimate dimer (see above). However, the polypeptide chains $A$ and $B$ do form crystal contacts (Fig. 5) that, according to PISA (Krissinel \& Henrick, 2007), 'are not strongly indicative of complex formation in solution'. These contacts bury $<800 \AA^{2}$ of surface area per monomer (for reference, HIV-1 PR dimerization buries $\sim 1700 \AA^{2}$ per monomer) and are formed in a mutual fashion by interactions of the flap loop with loop-80 (Pro86-Val90). Loop-80 is an important element of the retropepsin structure as it participates in shaping the inhibitor-binding cavity. Another discernible mode of crystal packing, involving an $a$-translated molecule $B$, buries $\sim 400 \AA^{2}$ in contacts that are formed nearly exclusively by the flap loops. This is an intriguing observation because in dimeric retropepsins the flaps also contribute to protomer interactions (in addition to the $\mathrm{N}$ - and $\mathrm{C}$-termini and the active-site loops), especially in complexes, when they are lowered onto the bound inhibitor. In general, the lattice contacts in the present structure tend to shield from solvent the face of the molecule that is normally buried upon dimerization. When a dimer of HIV-1 PR (PDB entry 3hvp; Wlodawer et al., 1989) is superposed on molecule $A$ of the present structure, it is obvious that the crystal-lattice aggregates of M-PMV PR are different from the functional retropepsin dimer. In particular, the active-site loops, which in the homodimer are closely associated through a 'fireman's grip' and a water-mediated (or hydroxyl-mediated) contact between the catalytic aspartates, are far apart, with the $\mathrm{C}^{\alpha} \ldots \mathrm{C}^{\alpha}$ distance between the Asn 26 residues being $11.4 \AA$. It is evident from Fig. 5 that the monomers forming the crystallographic aggregates of M-PMV PR remain associated by the flaps but are 'pulled apart' in the active site and $\mathrm{N}$-/C-terminal areas. In other words, the protomers are in close proximity and quite well juxtaposed for productive association but still do not interdigitate their $\mathrm{N}$-/C-termini in a proper dimeric association. One might speculate that the dimer does not assemble by side-by-side alignment of pre-formed monomeric proteins but is more likely to arise during the folding process that involves the formation of the dimer interface (from the $\mathrm{N}$ - and C-termini) at an early rather than late stage, as observed for HIV-1 PR (Ishima et al., 2001).

\section{Conclusions and outlook}

The present structure shows that retroviral protease can fold and exist stably as a monomer. This lends support to the notion of using dimerization inhibitors as potential antiretroviral drugs. The disruption of the dimeric interface might, for instance, be achieved by complexing the protein with an oligopeptide with the $\mathrm{N}$ - and $\mathrm{C}$-terminal sequences. In the case of M-PMV PR this should be even easier because one might exploit the potential to form a protease-inhibitor $\mathrm{S}-\mathrm{S}$ bond, but a similar strategy would also be possible for HIV-1 PR, which contains a Cys95 residue at the C-terminus.

We are grateful to Dr Richard Hrabal for providing energyminimized models of the NMR structure 1nso and to Martin
Hradilek for synthesizing the inhibitor. We acknowledge the Foldit players who contributed to the solution of this crystal structure. This work was supported by the Center for Game Science, DARPA grant N00173-08-1-G025, the DARPA PDP program, NSF grants IIS0811902 and IIS0812590, and by the Howard Hughes Medical Institute (DB). The material is based in part upon work supported by the National Science Foundation under Grant No. 0906026. This work was supported in part by grants 1-M0508 and Z40550506 from the Czech Ministry of Education to IP.

\section{References}

Adams, P. D. et al. (2010). Acta Cryst. D66, 213-221.

Baker, N. A., Sept, D., Joseph, S., Holst, M. J. \& McCammon, J. A. (2001). Proc. Natl Acad. Sci. USA, 98, 10037-10041.

Bauerová-Zábranská, H., Stokrová, J., Strísovsky, K., Hunter, E., Ruml, T. \& Pichová, I. (2005). J. Biol. Chem. 280, 42106-42112.

Cohen, G. H. (1997). J. Appl. Cryst. 30, 1160-1161.

Cooper, S., Khatib, F., Treuille, A., Barbero, J., Lee, J., Beenen, M., Leaver-Fay, A., Baker, D. \& Popović, Z. (2010). Nature (London), 466, 756-760.

DeLano, W. L. (2002). PyMOL. http://www.pymol.org.

DiMaio, F., Terwilliger, T. C., Read, R. J., Wlodawer, A., Oberdorfer, G., Wagner, U., Valkov, E., Alon, A., Fass, D., Axelrod, H. L., Das,

D., Vorobiev, S. M., Iwaï, H., Pokkuluri, P. R. \& Baker, D. (2011). Nature (London), 473, 540-543.

Emsley, P. \& Cowtan, K. (2004). Acta Cryst. D60, 2126-2132.

French, S. \& Wilson, K. (1978). Acta Cryst. A34, 517-525.

Hartl, M. J., Wöhrl, B. M., Rösch, P. \& Schweimer, K. (2008). J. Mol. Biol. 381, 141-149.

Ishima, R., Ghirlando, R., Tözsér, J., Gronenborn, A. M., Torchia, D. A. \& Louis, J. M. (2001). J. Biol. Chem. 276, 49110-49116.

Jancarik, J. \& Kim, S.-H. (1991). J. Appl. Cryst. 24, 409-411.

Jaskólski, M., Miller, M., Rao, J. K., Leis, J. \& Wlodawer, A. (1990). Biochemistry, 29, 5889-5898.

Kabsch, W. (2010). Acta Cryst. D66, 125-132.

Kabsch, W. \& Sander, C. (1983). Biopolymers, 22, 2577-2637.

Kervinen, J., Lubkowski, J., Zdanov, A., Bhatt, D., Dunn, B. M., Hui, K. Y., Powell, D. J., Kay, J., Wlodawer, A. \& Gustchina, A. (1998). Protein Sci. 7, 2314-2323.

Khatib, F., DiMaio, F., Foldit Contenders Group, Foldit Void Crushers Group, Cooper, S., Kazmierczyk, M., Gilski, M., Krzywda, S., Zabranska, H., Pichova, I., Thompson, J., Popovic, Z., Jaskolski, M. \& Baker, D. (2011). Nature Struct. Mol. Biol., doi:10.1038/ nsmb.2119.

Koh, Y., Matsumi, S., Das, D., Amano, M., Davis, D. A., Li, J., Leschenko, S., Baldridge, A., Shioda, T., Yarchoan, R., Ghosh, A. K. \& Mitsuya, H. (2007). J. Biol. Chem. 282, 2870928720.

Krissinel, E. \& Henrick, K. (2004). Acta Cryst. D60, 22562268.

Krissinel, E. \& Henrick, K. (2007). J. Mol. Biol. 372, 774-797.

Li, M., DiMaio, F., Zhou, D., Gustchina, A., Lubkowski, J., Dauter, Z., Baker, D. \& Wlodawer, A. (2011). Nature Struct. Mol. Biol. 18, 227-229.

Li, M., Laco, G. S., Jaskolski, M., Rozycki, J., Alexandratos, J., Wlodawer, A. \& Gustchina, A. (2005). Proc. Natl Acad. Sci. USA, 102, 18332-18337.

Miller, M., Jaskólski, M., Rao, J. K., Leis, J. \& Wlodawer, A. (1989). Nature (London), 337, 576-579.

Painter, J. \& Merritt, E. A. (2006). J. Appl. Cryst. 39, 109-111.

Parker, S. D. \& Hunter, E. (2001). Proc. Natl Acad. Sci. USA, 98, 14631-14636.

Svec, M., Bauerová, H., Pichová, I., Konvalinka, J. \& Strísovský, K. (2004). FEBS Lett. 576, 271-276. 


\section{research papers}

Veverka, V., Bauerová, H., Zábranský, A., Lang, J., Ruml, T., Pichová, I. \& Hrabal, R. (2003). J. Mol. Biol. 333, 771780.

Winn, M. D. et al. (2011). Acta Cryst. D67, 235-242.

Wlodawer, A., Miller, M., Jaskólski, M., Sathyanarayana, B. K., Baldwin, E., Weber, I. T., Selk, L. M., Clawson, L., Schneider, J. \&
Kent, S. B. (1989). Science, 245, 616-621.

Zábranská, H., Tůma, R., Kluh, I., Svatos, A., Ruml, T., Hrabal, R. \& Pichová, I. (2007). J. Mol. Biol. 365, 1493-1504.

Zábranský, A., Andreánsky, M., Hrusková-Heidingsfeldová, O., Havlícek, V., Hunter, E., Ruml, T. \& Pichová, I. (1998). Virology, 245, 250-256. 